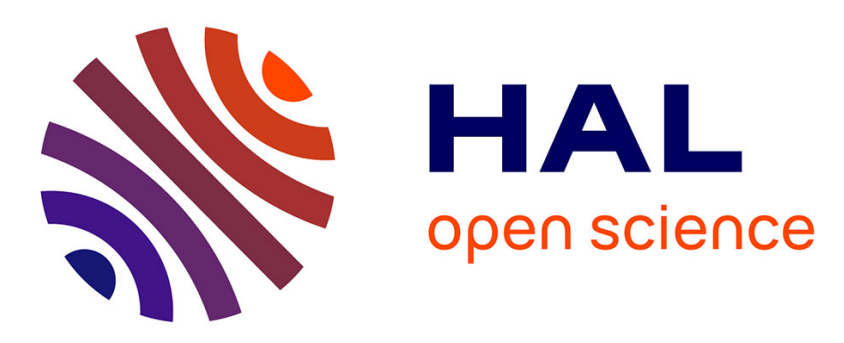

\title{
Competition and coexistence of ultrashort pulses in passive mode-locked lasers under dissipative-soliton-resonance conditions
}

Andrey Komarov, Foued Amrani, Alexander Dmitriev, Konstantin Komarov, François Sanchez

\section{To cite this version:}

Andrey Komarov, Foued Amrani, Alexander Dmitriev, Konstantin Komarov, François Sanchez. Competition and coexistence of ultrashort pulses in passive mode-locked lasers under dissipative-solitonresonance conditions. Physical Review A : Atomic, molecular, and optical physics [1990-2015], 2013, 87 (2), pp.023838. 10.1103/PhysRevA.87.023838 . hal-03204236

\section{HAL Id: hal-03204236 \\ https://univ-angers.hal.science/hal-03204236}

Submitted on 21 Apr 2021

HAL is a multi-disciplinary open access archive for the deposit and dissemination of scientific research documents, whether they are published or not. The documents may come from teaching and research institutions in France or abroad, or from public or private research centers.
L'archive ouverte pluridisciplinaire HAL, est destinée au dépôt et à la diffusion de documents scientifiques de niveau recherche, publiés ou non, émanant des établissements d'enseignement et de recherche français ou étrangers, des laboratoires publics ou privés. 


\title{
Competition and coexistence of ultrashort pulses in passive mode-locked lasers under dissipative-soliton-resonance conditions
}

\author{
Andrey Komarov, ${ }^{1,2, *}$ Foued Amrani, ${ }^{3}$ Alexander Dmitriev, ${ }^{2}$ Konstantin Komarov, ${ }^{1}$ and François Sanchez ${ }^{3}$ \\ ${ }^{1}$ Institute of Automation and Electrometry, Russian Academy of Sciences, Acad. Koptyug Pr. 1, 630090 Novosibirsk, Russia \\ ${ }^{2}$ Novosibirsk State Technical University, K. Marx Pr. 20, 630092 Novosibirsk, Russia \\ ${ }^{3}$ Laboratoire de Photonique d'Angers EA 4644, Université d'Angers, 2 Bd. Lavoisier, 49000 Angers, France
}

(Received 28 December 2012; published 25 February 2013)

\begin{abstract}
It has been shown by numerical simulation that the dissipative soliton resonance suppresses the appearance of new ultrashort pulses in the laser cavity that usually arise with increasing pumping. As a consequence, the energy of the pulses can reach an arbitrarily large value, which is determined by the corresponding pump power. The mechanism of the suppression is due to interaction between pulses through a saturable gain medium. The generation remains multistable: the number of pulses in steady-state operation depends on the initial conditions. In the case of multipulse generation, all pulses have identical durations, shapes, peak intensities, and chirps. The effect of maintaining single pulse operation with increasing pump power can be used to generate high-energy pulses.
\end{abstract}

DOI: 10.1103/PhysRevA.87.023838

PACS number(s): 42.65.Tg, 42.65.Sf, 42.60.Fc

\section{INTRODUCTION}

Passive mode-locked fiber lasers are widely used in various areas of science, technology, and engineering. These lasers have unique capabilities. They are reliable, compact, and flexible and can be conveniently pumped with commercially available semiconductor lasers. The nonlinear losses producing ultrashort pulses in such generators can be obtained using the nonlinear polarization rotation technique. These losses are fast, practically inertia free, and suitable for producing femtosecond pulses. A great variety of operating regimes is an important advantage of this type of laser. They can operate with a single pulse in a laser cavity or with various multipulse structures [1,2]. The properties of these structures are determined by the nature of interpulse interaction. Multipulse operation is due to the effect of quantization of the intracavity lasing radiation into individual identical solitons [3]. Multipulse structures can be generated in the form of a two-soliton molecule with different phase differences for the peak amplitudes of the solitons [4-7], multisoliton information sequences [8], and multisoliton complexes analogous to the different states of aggregation of matter: a soliton gas, a liquid, a glass, a soliton crystal [9], and so on. The long-range repulsion of ultrashort pulses in a laser cavity leads to harmonic passive mode locking [10-12] (a multipulse generation regime in which the distances between all neighboring pulses are identical). Harmonic passive mode-locked fiber lasers are of great interest as sources of ultrashort optical pulses with a high repetition rate, which are employed in high-speed optical communications and various femtosecond technologies. In this case, the multipulse generation due to the quantization of the intracavity lasing radiation into individual identical solitons is a very useful effect: the greater the number of pulses in a laser cavity, the higher the pulse repetition rate in the output radiation.

There has been considerable research activity in developing fiber generators of high-energy pulses [13-15]. The key

\footnotetext{
*Corresponding author: komarov@iae.nsk.su
}

obstacle for the generation of such pulses is the abovementioned multipulse operation due to the quantization of radiation into individual solitons. Indeed, due to this phenomenon, increasing the pumping results in an increase in the number of intracavity pulses, so that the energy of an individual pulse remains almost unchanged. In this case, the radiation quantization effect is a very harmful phenomenon that prevents an increase in the energy of an individual pulse with increasing pumping. Suppression of multipulse operation for obtaining a lasing regime with a single pulse in the laser resonator provides new opportunities for increasing the pulse energy.

In one study [16], conditions for single pulse operation are analyzed for a simple model of laser passive mode locking. This model assumes that the dispersions of the gain, losses, and refractive index are quadratic and the nonlinear losses and the nonlinear refractive index are proportional to the radiation intensity. It is shown that, in this case, passive mode locking occurs only in the form of single pulse operation. There is also a large area of nonlinear-dispersion parameters for which passive mode locking is not achieved. In this case, the radiation completely fills the laser resonator. Furthermore, there are solutions with an infinite increase in field intensity over time. However, these solutions are physically incorrect and are related to the imperfection of the model for the nonlinear losses proportional to radiation intensity. Indeed, in real experimental systems, decreases in losses cannot be greater than linear losses. Models that take into account this factor lead to multipulse operation at high pumping levels [3].

The authors of [17-20] used a model of nonlinear losses described by an additional term with a quadratic dependence on intensity. In this case, an infinite increase in intensity is suppressed due to this dependence. It was found that within the framework of this model with certain nonlineardispersion parameters the pulse shape became rectangular and its energy was arbitrarily high. This phenomenon was termed a dissipative soliton resonance, and it is of interest for designing high-energy pulse lasers [19]. Conditions for the occurrence of a single pulse and multipulse operation were not studied. Interaction of pulses through a common gain medium was ignored. 
In this paper we investigate the multipulse operation, competition, and coexistence of ultrashort laser pulses under dissipative soliton resonance conditions. These processes are due to the interaction of the pulses through their common gain medium. They determine the type of the steady-state passive mode-locking regime. In Sec. II we present a laser model for the study of the competition and coexistence of pulses under dissipative soliton resonance conditions. Numerical simulations and the results are discussed in Sec. III. The main conclusions are given in Sec. IV.

\section{MODEL}

For our analysis we use the following master equation describing the field evolution in a unidirectional ring laser based on the model of a distributed intracavity medium with a quadratic complex dispersion and a nonlinear refractive index and nonlinear losses [21,22]:

$$
\frac{\partial E}{\partial \zeta}=\left(D_{r}+i D_{i}\right) \frac{\partial^{2} E}{\partial \tau^{2}}+\left[g+(p+i q) I-\left(p_{2}+i q_{2}\right) I^{2}\right] E,
$$

where $E(\zeta, \tau)$ is the electric-field amplitude; $\tau$ is the time coordinate in units $\delta t=\sqrt{\left|\beta_{2}\right| L / 2}$ (here $\beta_{2}$ is the second-order group-velocity dispersion for the intracavity medium and $L$ is the cavity length); $\zeta$ is the normalized propagation distance (the number of passes of the radiation through the laser cavity); $D_{r}$ and $D_{i}$ are the frequency dispersions of the gain losses and the refractive index, respectively; $q$ and $q_{2}$ are the Kerr nonlinearities; and $I=|E|^{2}$ is the field intensity. The term $g$ is the total amplification including the linear losses $\sigma_{0}$ :

$$
g=\frac{a}{1+b \int I d \tau}-\sigma_{0},
$$

where the integration is carried out over the whole round-trip period, $a$ is the pumping parameter, and $b$ is the saturation parameter. The term $p I$ describes nonlinear losses that decrease with increasing intensity $I$. The term $p_{2} I^{2}$ is due to nonlinear losses that increase with increasing $I$. In the case of such nonlinear losses, the growth in the peak intensity of pulses is limited by the value $I_{\max } \sim p / p_{2}$. A similar limitation of the peak intensity occurs in passive mode-locked fiber lasers with nonlinear losses due to the nonlinear polarization rotation technique [2]. Equation (1) takes into account the gain saturation through the dependence of the parameter $g$ on the energy of the intracavity radiation [see Eq. (2)]. This differentiates Eq. (1) from the equation used in [5,18-21], where the parameter $g$ is a constant.

The scalar model described by Eq. (1) gives temporal and spectral profiles of a stationary single pulse that are qualitatively similar to the corresponding results obtained using a vector model of passive mode locking for fiber lasers [2] (see Sec. III). At the same time, this simpler model provides a better understanding of the various features of passive mode locking. These factors determine the use of the model Eq. (1) for the analysis of the investigated regimes of passive mode locking of fiber lasers.

\section{NUMERICAL SIMULATIONS AND DISCUSSION}

In the case of the cubic nonlinearity alone $\left(p_{2}=0, q_{2}=0\right)$ Eq. (1) has the following analytical solution for the temporal profile of a stationary pulse [22]:

$$
E_{s}=E_{0} \frac{\exp (i \delta k \zeta)}{\cosh ^{1+i \alpha} \beta \tau}
$$

Here the peak amplitude of the pulse $E_{0}$, the inverse of the pulse duration $\beta$, the pulse frequency chirp $\alpha$, and the parameter $\delta k$ are determined from a system of algebraic equations (see the Appendix). The chirp is given by the equation

$$
\frac{\alpha}{2-\alpha^{2}}=\frac{\xi-\theta}{3(1+\xi \theta)}
$$

where $\xi=q / p$ and $\theta=D_{i} / D_{r}$. Figure 1(a) shows the change in the chirp $\alpha$ on the plane of the nonlinear-dispersion parameters $\xi$ and $\theta$. The spectral profile of the stationary pulse
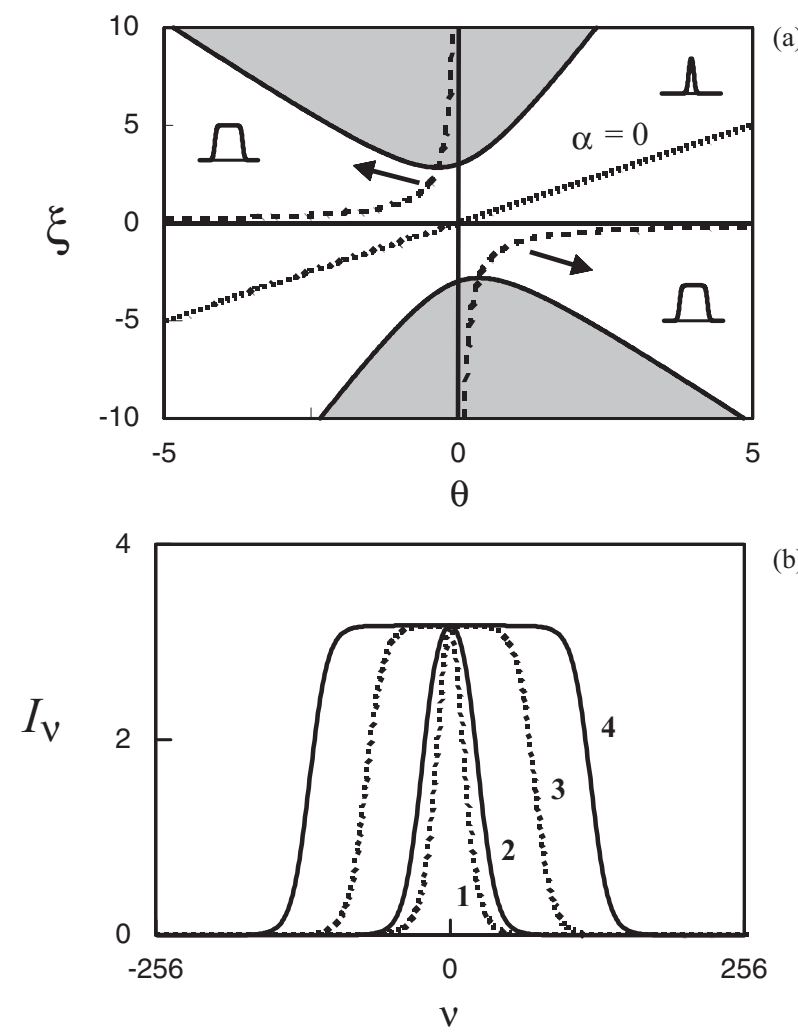

(b)

FIG. 1. Changes in the spectrum of a stationary ultrashort pulse described by Eq. (1) with the complex cubic nonlinearity $\left(p_{2}=0\right.$, $q_{2}=0$ ). (a) Variation of the chirp $\alpha$ in the plane of the nonlineardispersion parameters $\xi=q / p, \theta=D_{i} / D_{r}$. For the dotted line $(\xi=$ $\theta)$, the stationary pulse is spectrally limited $(\alpha=0)$; for the dotted curves $(\xi=-1 / \theta) \alpha= \pm \sqrt{2}$, the arrows point the directions of the maximum increase in the chirp $\alpha$ where the the spectral profiles take a rectangular shape. For the gray areas, passive mode locking does not occur. The boundary solid curves are determined from the condition $g(\xi, \theta)=0$. (b) The spectral profiles of steady-state solitons with different chirps: (1) $\alpha=0$, (2) $\alpha=1$, (3) $\alpha=3$, and (4) $\alpha=5$. 
Eq. (3) is given by the following analytical expression [22]:

$$
I_{\nu}=\frac{\pi^{2}\left|E_{0}\right|^{2}}{\beta^{2}} \frac{\sinh \pi \alpha}{\alpha\left(\cosh \pi \alpha+\cosh \frac{\pi v}{\beta}\right)},
$$

where $v$ is the frequency detuning from the center carrier frequency of the soliton. With increasing chirp $\alpha$, the spectral profile changes from a bell-shaped to a rectangular form [see Fig. 1(b)].

The right upper quadrant in Fig. 1(a) corresponds to the focusing nonlinearity $(q>0)$ and the anomalous dispersion $\left(D_{i}>0\right)$ of the refractive index. The left upper quadrant corresponds to the focusing nonlinearity and the normal dispersion $\left(D_{i}<0\right)$ of the refractive index. For the nonlineardispersion parameters from the white area, steady-state passive mode locking occurs only with a single pulse in the laser cavity. The quantity $g$ describes the total amplification for the weak field outside the volume of the stationary pulse. For the white area, it is negative: $g<0$. For the nonlinear-dispersion parameters from the gray area, $g>0$ and the regime of the stationary single pulse is unstable. In this case, a lasing regime is established for which the laser cavity is completely filled with radiation. The condition $g=0$ is typical for a change in the lasing regime established after a transient process.

Using Eqs. (1) and (2) with $p_{2} \neq 0$, we studied the features of the passive mode locking observed with a limitation of a decrease of nonlinear losses with increasing intensity. Numerical simulation was performed with the focusing nonlinearity $q$ and various values of the normal dispersion $D_{i}$ [the left upper quadrant in Fig. 1(a), where a frequency chirp $\alpha$ can reach the greatest magnitude]. Plots of the number of intracavity pulses in steady-state operation $N$ versus pump power $a$ are presented in Fig. 2. The procedure used to construct the dependence $N=N(a)$ is as follows. The initial field was chosen in the form of several pulses of different amplitudes. This was done to model initial noise pulses. A steady state was established after a transient process. The number of pulses in the steady state was plotted in Fig. 2. Then pump power $a$ was changed slightly (with a step of 0.1 ) and, after a transient process, the number of pulses was plotted again, and so on. The dependence $N=N(a)$ was determined in this way. The variance of the pulse amplitudes due to spontaneous noise radiation was modeled by the addition of the initial multipulse field reduced by a factor of 100 to the generated radiation at the instant the pump power was changed. This perturbation accelerated the transient process in the vicinity of bifurcation points. To obtain steady-state multipulse operation in the case of Fig. 2(c), we chose a sufficiently large value of the pump power $a$ and a sufficiently small amplitude difference in the corresponding number of initial pulses. Thereafter the pump power was changed with a small step. In the case of steady-state multipulse operation, all pulses had identical parameters (shape, peak amplitude, and frequency chirp). An increase in the number of pulses is possible only in accordance with the lower stepwise curve marked by arrows in Fig. 2 . The number of pulses decreases in accordance with the upper stepwise curve.

Figure 3 demonstrates the dependence of the amplification $g$ on the pump power $a$. Here, as in Fig. 1(a), the condition $g=0$ determines the boundary separating different lasing

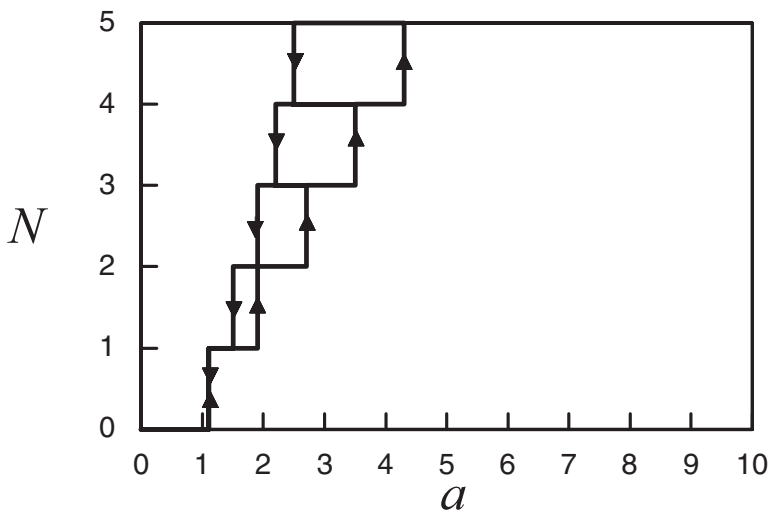

(a)

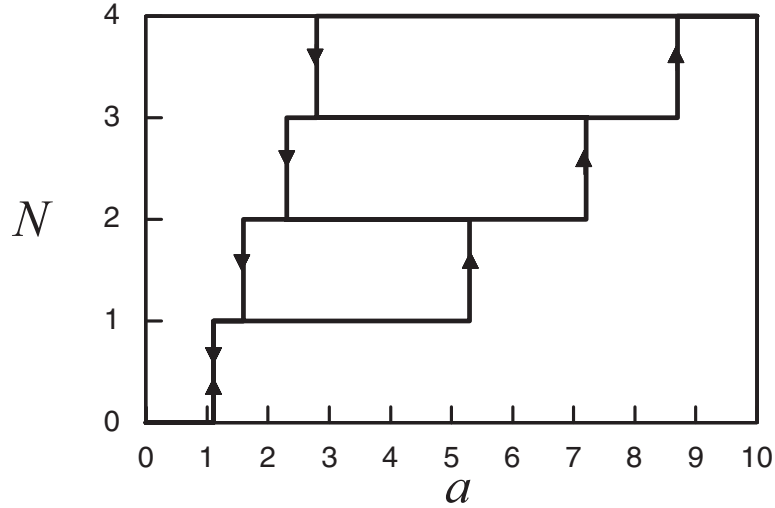

(b)

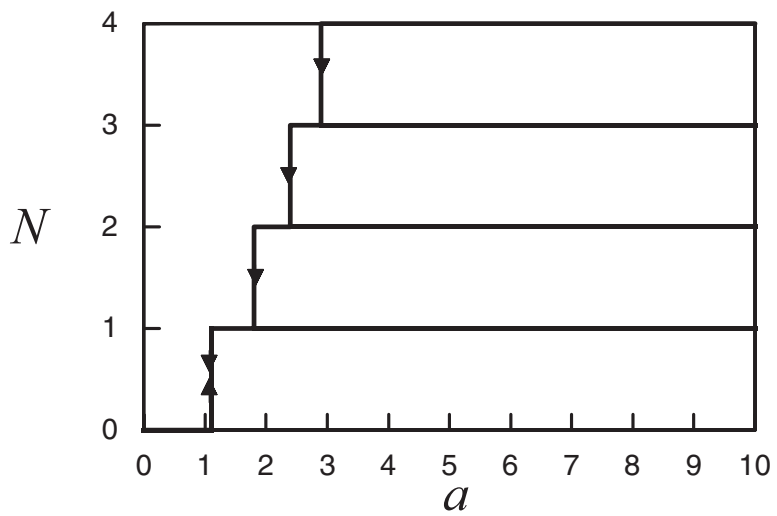

(c)

FIG. 2. Number of pulses $N$ vs pump power $a$. (a) $D_{i}=-20$. (b) $D_{i}=-25$. (c) $D_{i}=-28$. For all figures, $D_{r}=1, p=1, q=18$, $\sigma_{0}=1, p_{2}=3, q_{2}=0$, and $b=0.01$.

regimes (regimes with different numbers of pulses in the laser cavity). The jumps in Fig. 3(a) are in one-to-one correspondence with the jumps of the lower stepwise curve in Fig. 2(a). Such jumps occur when the amplification $g$ reaches a zero value and, as a result, a new pulse appears in the laser cavity. Increase in the amplification $g$ with increasing pump power $a$ is due to a drop of the efficiency of interaction of the pulse with the gain medium. This effect is related to the finite width of the gain frequency band and an increase in the spectral width of the pulse with increasing pulse peak intensity. The mechanism of appearance of new pulses in generation with increasing pump power and the physical nature of the quantization of radiation into individual identical solitons in passive mode-locked lasers were investigated and discussed in detail in previous papers $[2,3,16]$. In the analysis of these 


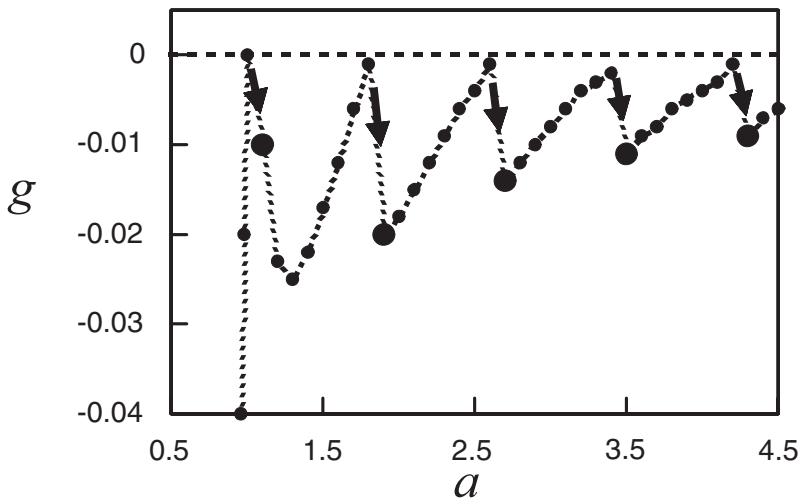

(a)

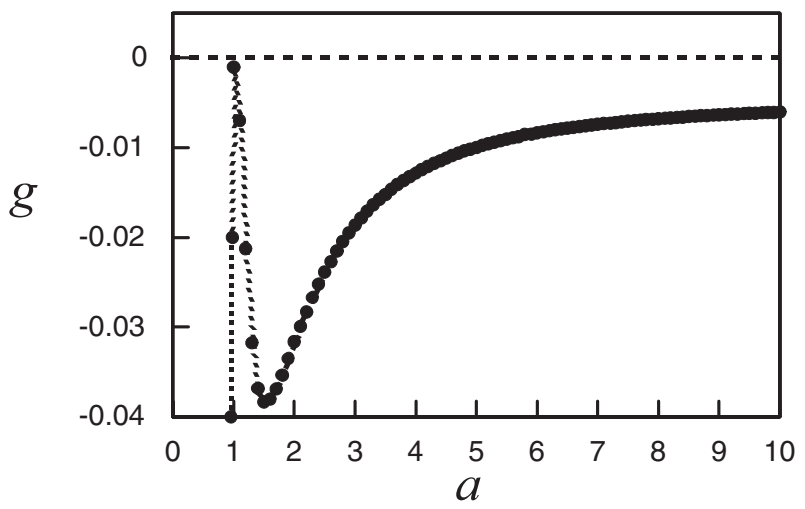

(b)

FIG. 3. Dependence of the total amplification $g$ on the pump power $a$. (a) Usual passive mode locking. The arrows point to jumplike transitions from steady states with $g=0$ to the state with $g<0$ designated by large black circles. $D_{i}=-20$. (b) Passive mode locking under dissipative soliton resonance conditions. The dependence $g=g(a)$ corresponds to the lowest branch in Fig. 2(c). $D_{i}=-28$. Other parameters are the same as in Fig. 2 .

phenomena, it is necessary to take into account the dependence of the amplification $g$ on the total energy of the intracavity radiation [see Eq. (2)].

Increase in the number of pulses in a laser cavity with increasing pump power $a$ is common for models of nonlinear losses taking into account that their decrease with increasing intensity $I$ is limited. However, as one can see from Fig. 2(c), in the case of a high normal dispersion $D_{i}$, this is not so. The single pulse operation regime is retained with increasing pumping. This regime is stable because the amplification $g$ outside the volume of the pulse remains negative as the pump power $a$ is increased [see Fig. 3(b)]. Figures 4 and 5 explain the nature of this phenomenon.

Figure 4 shows that with increasing pump power $a$ the peak intensity of the steady-state pulse initially increases and then remains constant while its bell-shaped form is transformed into a rectangular one. Further increase in the pumping $a$ results in a monotonic increase in the duration of the rectangular pulse. This transformation of the stationary pulse is associated with the stabilizing quadratic nonlinearity of the losses $p_{2}$ in Eq. (1). The corresponding change in the pulse spectrum is shown in Fig. 5. As the pump power $a$ is increased, a bell-shaped top appears in the rectangular spectral profile [see Fig. 5(a)]. Further increase in the pump power leads to the growth of the bell-shaped part of the spectrum, and the

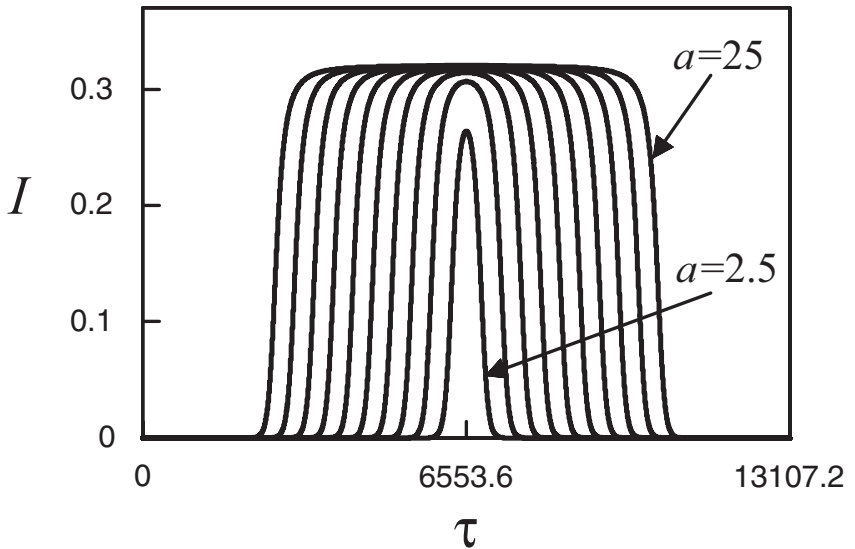

FIG. 4. Dependence of the temporal profile of a steady-state pulse on the pump power $a$. The pump power is changed with a step $\delta a=$ 2.5. Laser parameters are the same as in Fig. 3(b).

rectangular spectral profile is transformed in this way to a bell-shaped one [see Fig. 5(b)]. An important point is that the bell-shaped part of the spectrum is significantly narrower than the rectangular part. The rectangular part is due to the soliton wings, where high intensity gradients occur, resulting in a great frequency chirp, that is, the broadband rectangular portion of the spectrum. In contrast, in the central part of the rectangle pulse, the intensity gradients are small and, as a result, the frequency variation is also small. Accordingly, this part of the pulse is responsible for the narrowband bell-shaped portion of the spectrum. When the length of the central part of
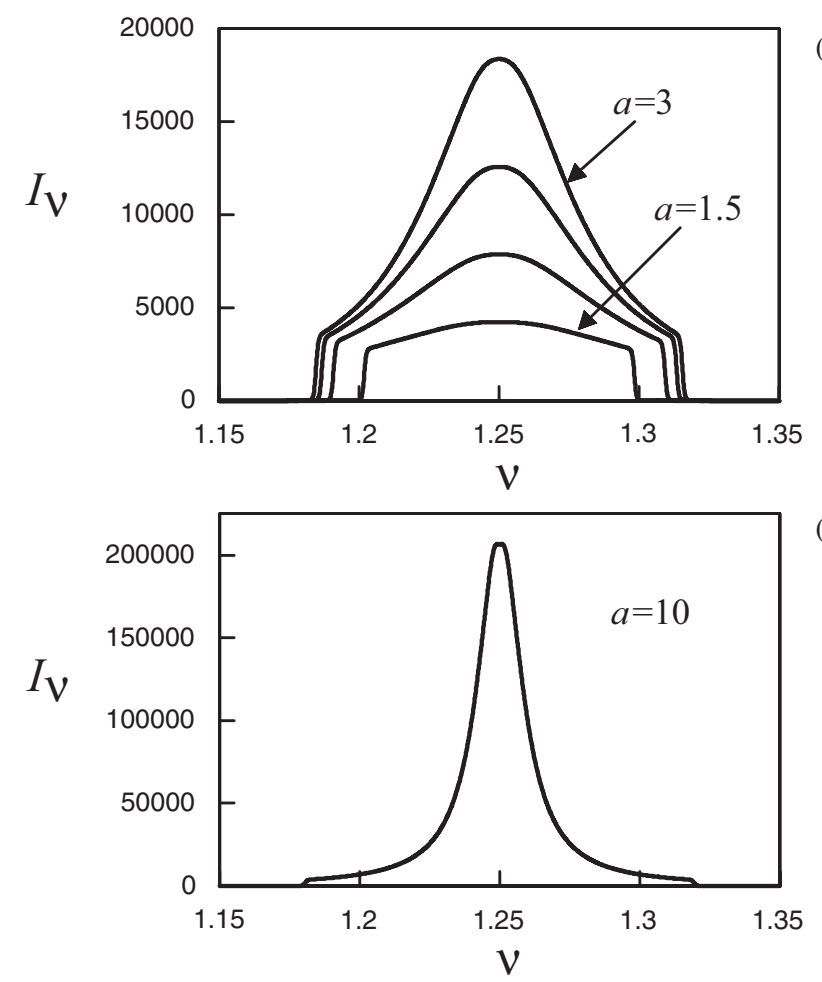

(b)

FIG. 5. Dependence of the spectral profile of a steady-state pulse on the pump power $a$. (a) The pump power changed with a step $\delta a=0.5$. (b) $a=10$. All other parameters are the same as in Fig. 4. 
the pulse becomes large in comparison with the length of the wings, the spectrum of the pulse is considerably narrowed [see Fig. 5(b)]. As a result, the detuning of the spectral components of the pulse from the center of the spectral gain band becomes considerably smaller, and the pulse interacts with the gain medium much more effectively. Thus, the term dissipative soliton resonance can be understood literally, that is, in the sense of such effective resonance interaction. As a result of this interaction, the inverse population in the gain medium is effectively depleted. The amplification $g$ outside the pulse becomes negative, $g<0$, which prevents the appearance of new pulses in the laser cavity from spontaneous radiation with increasing pump power. Accordingly, with increasing pump power $a$, the energy of a single pulse can be arbitrarily large at a corresponding level of pumping.

In the case of dissipative soliton resonance, passive modelocked lasers also show multistability [see Fig. 2(c)]: the number of stationary pulses in steady-state operation depends on the initial conditions. Operation with initial pulses differing in duration becomes steady-state operation with identical pulses. Single pulse operation is obtained only with a single initial pulse or with small initial pump power $a$. With increasing pump power, the single pulse operation is retained.

The phenomenon of dissipative soliton resonance is due to the specific dependence of nonlinear losses on the intensity $\delta \sigma=-p I+p_{2} I^{2}$. At low intensity, these losses decrease with increasing intensity. In contrast, at high intensity, they increase as the intensity is increased. As a result, in the case of dissipative soliton resonance, the peak intensity is stabilized at a certain level $I_{\max } \sim p / p_{2}$ and the pulse becomes rectangular. A similar dependence is observed in passive mode-locked fiber lasers with the nonlinear polarization rotation technique. Correspondingly, the analysis of the generation dynamics of these lasers based on the vector model also leads to rectangle pulses and the specific spectral dependence presented in Figs. 4 and 5 (see Fig. 4 in [2]). Thus, dissipative soliton resonance is a rather common phenomenon and can be observed in real lasers with passive mode locking. For the case of anomalous dispersion, this phenomenon was observed in [23]. The scalar model Eq. (1) adequately describes the phenomenon studied. It is significantly simpler than the vector model and provides a better understanding of the main features of the passive mode-locking process related to dissipative soliton resonance.

\section{CONCLUSION}

In this paper we developed a theoretical model to describe the competition and coexistence of ultrashort pulses in passive mode-locked lasers with dissipative soliton resonance. The model includes the quadratic dispersion of the gain, losses, and refractive index for the intracavity distributed medium, its Kerr nonlinearity, and the saturable gain dependent on the radiation energy. The nonlinear losses are described by a linear-quadratic dependence on the intensity. In the case of dissipative soliton resonance, the lasing pulses have a rectangular form. We have found that this form of the pulses results in their narrower spectrum and, hence, in their more effective interaction with the gain medium and more considerable saturation of the gain. In this case, the total amplification for the weak radiation outside the pulse volume becomes negative, which prevents the appearance of new pulses in the laser cavity with increasing pumping, and the number of generated pulses remains constant. As a result, with increasing pump power, the energy of a single pulse can be arbitrarily large at a corresponding level of pumping. In multipulse operation, all pulses have identical parameters (form, peak intensity, spectrum, and so on). The generation is multistable. In the case of dissipative soliton resonance, the number of pulses depends on the initial conditions, but it does not change with increasing pump power. Smaller nonlinearities of the refractive index and larger values of the normal dispersion promote dissipative soliton resonance. This phenomenon provides new opportunities for the design of high-energy pulse lasers.

\section{ACKNOWLEDGMENTS}

This work was supported by the Agence Nationale de la Recherche (Contract No. ANR-2010-BLANC-0417-01SOLICRISTAL) and the Russian Federation (RF) President's Grants No. MK-2602.2011.2 and No. NS-6170.2012.2.

\section{APPENDIX: PULSE SOLUTION OF THE CUBIC MASTER EQUATION}

Substituting the expression for the stationary pulse $E_{s}$ from Eq. (3) into Eq. (1) and separately equating the terms proportional to $E_{s}$ and $E_{s} / \cosh ^{2}(\beta \tau)$, we obtain the following algebraic equations for the parameters of the stationary pulse:

$$
\begin{gathered}
\left(D_{r}+i D_{i}\right)(1+i \alpha)^{2} \beta^{2}-i \delta k+g=0, \\
-\left(D_{r}+i D_{i}\right)(1+i \alpha)(2+i \alpha) \beta^{2}+(p+i q)\left|E_{0}\right|^{2}=0 .
\end{gathered}
$$

The complex algebraic equation (A2) corresponds to the two real equations

$$
\begin{aligned}
& D_{i}\left(2-\alpha^{2}\right) \beta^{2}+3 D_{r} \alpha \beta^{2}=q\left|E_{0}\right|^{2}, \\
& D_{r}\left(2-\alpha^{2}\right) \beta^{2}-3 D_{i} \alpha \beta^{2}=p\left|E_{0}\right|^{2} .
\end{aligned}
$$

Division of Eq. (A3) by Eq. (A4) yields Eq. (4), which defines the frequency chirp $\alpha$ through the nonlinear-dispersion parameters $\xi=q / p$ and $\theta=D_{i} / D_{r}$. As can be seen from this equation, in the case of the parameters $\xi$ and $\theta$ corresponding to the line $\xi=\theta$ [the dotted line in Fig. 1(a)], the chirp is equal to zero: $\alpha=0$. For the parameters $\xi$ and $\theta$ lying above (below) this straight line, the chirp is positive (negative). For the values of $\xi$ and $\theta$ lying on the dotted curve $\xi=-1 / \theta$, the chirp is equal to $\sqrt{2}$ for the left upper quadrant in Fig. 1(a), and it is equal to $-\sqrt{2}$ for the lower right quadrant [for $\alpha= \pm \sqrt{2}$ and $\xi=-1 / \theta$, the denominators of the right and left sides of Eq. (4) are equal to zero].

From the complex equation (A1) we obtain the following two real equations:

$$
\begin{aligned}
& \delta k=2 D_{r} \alpha \beta^{2}+D_{i}\left(1-\alpha^{2}\right) \beta^{2}, \\
& g=-D_{r}\left(2-\alpha^{2}\right) \beta^{2}+2 D_{i} \alpha \beta^{2} .
\end{aligned}
$$

For known values of $\alpha$ and $\beta$ Eq. (A5) allows one to determine the correction $\delta k$ for the central wave vector of the pulse. 
The chirp $\alpha$ is determined from Eq. (4). The peak amplitude $E_{0}$, the amplification $g$, and the inverse of the duration $\beta$ are determined from the system of algebraic equations containing Eqs. (A6) and (2) in the form

$$
g=\frac{a}{1+2 b\left|E_{0}\right|^{2} / \beta}-\sigma_{0}
$$

and the system containing Eqs. (A3) and (A4).

A necessary condition for the stability of the stationary pulse Eq. (3) is the condition $g<0$. Otherwise, any perturbations outside the stationary pulse will be built up with time. Equation (A6) with $g=0$ and Eq. (4) define the boundary $\xi=\xi(\theta)$ separating the regions Of stability and instability of the stationary pulse Eq. (3) on the plane $\xi, \theta$ shown in Fig. 1(a):

$$
\xi= \pm \frac{3 \sqrt{1+\theta^{2}} \mp 2 \theta+2 \theta^{2}\left(\sqrt{1+\theta^{2}} \mp \theta\right)}{1 \mp \theta\left(\sqrt{1+\theta^{2}} \mp \theta\right)} .
$$

The upper sign corresponds to the upper solid curve in the figure. The lower sign corresponds to the lower solid curve. The perturbations outside the stationary pulse are suppressed due to the negative value of $g$. The energy of the pulse is stabilized due to the saturation of the amplification $g$, which depends on the pulse energy. Outside the area bounded by the solid curves in Fig. 1(a), the amplification is positive: $g>0$. As a result, the stationary pulse is unstable, and after a transient process the laser cavity is completely filled with radiation.
[1] D. Y. Tang, L. M. Zhao, B. Zhao, and A. Q. Liu, Phys. Rev. A 72, 043816 (2005).

[2] A. Komarov, H. Leblond, and F. Sanchez, Phys. Rev. A 71, 053809 (2005).

[3] A. K. Komarov and K. P. Komarov, Phys. Rev. E 62, R7607 (2000).

[4] B. A. Malomed, Phys. Rev. A 44, 6954 (1991).

[5] N. N. Akhmediev, A. Ankiewicz, and J.-M. Soto-Crespo, Phys. Rev. Lett. 79, 4047 (1997).

[6] D. Y. Tang, W. S. Man, H. Y. Tam, and P. D. Drummond, Phys. Rev. A 64, 033814 (2001).

[7] Ph. Grelu, F. Belhache, F. Gutty, and J. M. Soto-Crespo, Opt. Lett. 27, 966 (2002).

[8] A. Komarov, K. Komarov, and F. Sanchez, Phys. Rev. A 79, 033807 (2009).

[9] F. Amrani, A. Haboucha, M. Salhi, H. Leblond, A. Komarov, and F. Sanchez, Appl. Phys. B 99, 107 (2010).

[10] A. B. Grudinin and S. Gray, J. Opt. Soc. Am. B 14, 144 (1997).

[11] G. Sobon, K. Krzempek, P. Kaczmarek, K. M. Abramski, and M. Nikodem, Opt. Com. 284, 4203 (2011).

[12] Z. X. Zhang, L. Zhan, X. X. Yang, S. Y. Luo, and Y. X. Xia, Laser Phys. Lett. 4, 592 (2007).
[13] W. H. Renninger, A. Chong, and F. W. Wise, Opt. Lett. 33, 3025 (2008).

[14] E. J. R. Kelleher, J. C. Travers, E. P. Ippen, Z. Sun, A. C. Ferrari, S. V. Popov, and J. R. Taylor, Opt. Lett. 34, 3526 (2009).

[15] M. Erkintalo, C. Aguergaray, A. Runge, and N. G. R. Broderick, Opt. Express 20, 22669 (2012).

[16] A. K. Komarov and K. P. Komarov, Opt. Comm. 183, 265 (2000).

[17] N. N. Akhmediev, J.-M. Soto-Crespo, and Ph. Grelu, Phys. Lett. A 372, 3124 (2008).

[18] W. Chang, A. Ankiewicz, J.-M. Soto-Crespo, and N. N. Akhmediev, Phys. Rev. A 78, 023830 (2008).

[19] W. Chang, J.-M. Soto-Crespo, A. Ankiewicz, and N. N. Akhmediev, Phys. Rev. A 79, 033840 (2009).

[20] P. Grelu, W. Chang, A. Ankiewicz, J.-M. Soto-Crespo, and N. N. Akhmediev, J. Opt. Soc. Am. B 79, 2336 (2010)

[21] N. N. Akhmediev, V. V. Afanasjev, and J.-M. Soto-Crespo, Phys. Rev. E 53, 1190 (1996).

[22] K. P. Komarov, Opt. Spectrosc. 60, 231 (1986); 60, 379 (1986).

[23] L. Duan, X. Liu, D. Mao, L. Wang, and G. Wang, Opt. Express 20, 265 (2011). 